\title{
On the use of LSPIV-based free software programs for the monitoring of river: testing the PIVlab and the FUDAA-LSPIV with synthetic and real image sequences
}

Dario Pumo, Francesco Alongi, Giuseppe Ciraolo, and Leonardo Noto

Dipartimento di Ingegneria, Università degli Studi di Palermo, Palermo, Italy (dario.pumo@unipa.it)

The development of new image-based techniques is allowing a radical change in the environmental monitoring field. The fundamental characteristics of these methods are related to the possibility of obtaining non-intrusive measurements even in adverse circumstances, such as high flow conditions, which may seriously threaten the operators' safety conditions in traditional approaches.

Optical techniques, based on the acquisition, analysis and elaboration of sequences of images acquired by digital cameras, are aimed at a complete characterization of the river instantaneous surface velocity field, through the analysis of a floating tracer, which may be naturally present or artificially introduced. The growing availability of a new generation of both low-cost optical sensors and high-performing free software programs for image processing, is a key aspect explaining the rapid development of such techniques in recent years. The best known optical techniques are the large scale particle velocimetry (LSPIV) and the large scale particle tracking velocimetry (LSPTV).

This work is aimed to analyze and compare the performance of the two most common free software packages based on LSPIV (i.e. the PIVlab and the FUDAA-LSPIV), which use different crosscorrelation algorithms. The test is carried out by analyzing several sequences of both synthetic images and real frames acquired on natural rivers under different environmental conditions (with tracers artificially introduced). An image sequences generator has been implemented ad-hoc with the aim to create, under fixed configurations, synthetic sequences of images, simulating uniformly distributed tracers moving under controlled conditions. The various configurations are characterized by different parameterization in terms of: (i) flow velocity ( $\mathrm{S}=\mathrm{slow}$ or $\mathrm{F}=$ fast flow conditions, according to a logarithmic transverse flow profile); (ii) tracer particles size (CON= disks of constant diameter; VAR=disks of variable size with given mean diameter); (iii) seeding density per frame (density: low -LD, medium -MD, high -HD).

The synthetic sequences are processed by the two software packages together with the real sequences, analyzing the errors in terms of mean value of the surface velocity field and velocity along a transverse transect, with respect to a benchmark velocity (i.e. that imposed in the image sequence generator for the synthetic sequences and that deriving from the use of traditional sensors, i.e. ADCP, for the real sequences). 
